\title{
Transgenic Zebrafish as a Novel Animal Model to Study Tauopathies and Other Neurodegenerative Disorders in vivo
}

\author{
Dominik Paquet Bettina Schmid Christian Haass \\ Deutsches Zentrum für Neurodegenerative Erkrankungen - Standort München and Adolf-Butenandt-Institute, \\ Department of Biochemistry, Ludwig-Maximilians University, Munich, Germany
}

\section{Key Words}

Zebrafish • Gal4-UAS · Tau • Alzheimer's disease •

Frontotemporal dementia $\cdot$ Drug screening

\begin{abstract}
Our ageing society is confronted with a dramatic increase in patients suffering from tauopathies such as Alzheimer's disease, frontotemporal dementia and others. Typical neuropathological lesions including tangles composed of hyperphosphorylated tau protein as well as severe neuronal cell death characterize these disorders. No mechanism-based cures are available at present. Genetically modified animals are invaluable models to understand the molecular disease mechanisms and to screen for modifying compounds. We recently introduced tau-transgenic zebrafish as a novel model for tauopathies. Our model allows recapitulating key pathological features of tauopathies within an extremely short time. Moreover, life imaging of tau-dependent neuronal cell death was performed for the very first time. This demonstrated tau-dependent neuronal cell loss independent of tangle formation. Finally, we exemplified that the zebrafish frontotemporal dementia model can be used to screen for drugs that prevent abnormal tau phosphorylation and neuronal cell death.

Copyright ๑ 2010 S. Karger AG, Basel
\end{abstract}

Neurodegenerative diseases including Alzheimer's disease (AD) and frontotemporal dementia (FTD) are the most frequent cause of dementia in our ageing society. These disorders are characterized by posttranslationally modified amyloidogenic proteins, such as amyloid $\beta$ peptide $(A \beta)$ in $A D$ and tau in $A D$ and FTD, which form neurotoxic oligomers and are finally deposited as insoluble amyloid fibers in senile plaques or tangles [1-3].

Neurodegenerative diseases with deposition of insoluble tau protein are now commonly termed tauopathies. The tau protein is an important target for drug development, since its pathological alterations correlate with disease progression and memory loss in $\mathrm{AD}$ and other tauopathies [4]. Moreover, tau suppression improves memory function in transgenic FTD models [5]. Furthermore, mutations in the tau gene, such as the Tau-P301L mutation, have been identified in patients with a genetically inherited form of autosomal dominant FTD [6]. Among the first pathological alterations of tau is its pathologic phosphorylation at multiple residues [2]. Subsequently, the hyperphosphorylated tau species become insoluble and form paired helical filaments, which are finally deposited in the cell bodies of neurons as neurofibrillary tangles (NFT). The biochemical and pathological properties of tau have been extensively studied both in vitro and in vivo. Over the last decade, several tau trans-

\section{KARGER}

Fax +41613061234 E-Mail karger@karger.ch www.karger.com
Christian Haass, $\mathrm{PhD}$

Deutsches Zentrum für Neurodegenerative Erkrankungen, Standort München Adolf-Butenandt-Institute, Department of Biochemistry

Ludwig-Maximilians University, Schillerstrasse 44, DE-80336 Munich (Germany)

Tel. +49 892180 75472, Fax +49 892180 415, E-Mail chaass@med.uni-muenchen.de 
Fig. 1. The translucent body of zebrafish larvae allows imaging of cellular and subcellular processes in high detail. Triple immunostaining of a 5-day-old tau-transgenic zebrafish, using antibodies against acetylated tubulin, showing neuronal projections in green, total tau in red and AT8-phosphorylated tau in blue.

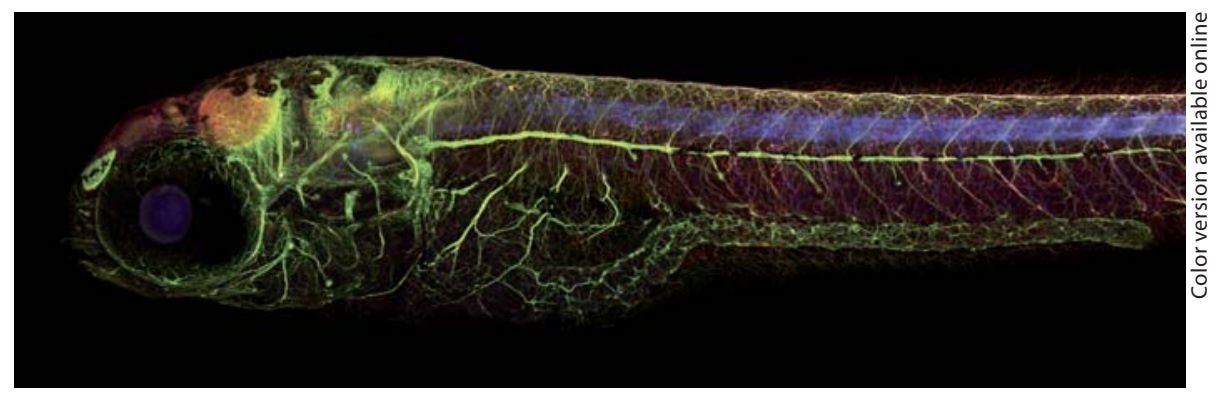

genic animal models have been developed. Invertebrates such as worms and flies and vertebrates like mice have been used to understand pathogenic mechanisms [for detailed reviews, see 7,8$]$. In addition, chemical modifiers of the disease which could be further explored to develop novel treatment approaches have been investigated in these animals. Invertebrate systems offer experimental advantages, including low costs, small size, excellent genetic tractability, a simple and rapidly developing nervous system and a short life span. However, the evolutionary and morphological distance between invertebrates and humans makes the direct translation of experimental results rather difficult and unreliable. Therefore, neurotoxic mechanisms evoked by amyloidogenic proteins may be more faithfully reproduced in vertebrate brains. For this reason, transgenic mice have been the major species used for modeling AD and FTD.

The generation of these mouse models began with the expression of wild-type tau, which led to its pathologic phosphorylation, but not filament formation. This key feature of tauopathies was only reproduced upon expressing tau with familial FTD-associated tau mutations. Subsequently, the FTD-like phenotypes could be further enhanced by the addition of $A \beta$, indicating cross-talk between $A \beta$ and tau aggregation. Finally, coexpression of mutant tau with the Swedish mutation of the $\beta$-amyloid precursor protein as well as the familial AD-associated presenilin $1 \mathrm{M} 146 \mathrm{~V}$ mutation, led to the enhanced formation of NFTs, again supporting the amyloid cascade hypothesis. However, tau seems to be the primary mediator of toxicity and memory loss, since inhibition of tau expression in such triple transgenic mice allowed recovery of memory and a reduction in neuronal cell loss. Interestingly, under these conditions NFTs continued to accumulate, suggesting that soluble tau oligomers but not tangles may be the primary neurotoxic species $[1,7,8]$.

The abovementioned models greatly expanded our knowledge on the formation of the typical neuropathol- ogy of AD and FTD and the pathologic misfunction of disease-associated genes. However, the initial steps leading to tau phosphorylation and synaptic dysfunction, which in humans may occur presymptomatically are still poorly understood in vivo. It is extremely difficult to investigate early subtle changes and disease propagation in living mice, since their brain is not readily accessible for imaging and they need a rather long time to develop disease symptoms. In addition, large-scale screening for anti-aggregation drugs, or neurotoxicity-ameliorating compounds, is not feasible in mice. Transgenic zebrafish larvae combine many of the advantages of invertebrate and vertebrate models. Their translucent body allows whole-body imaging of cellular and subcellular processes in high detail (fig. 1) both in fixed animals and in vivo [9]. In addition, large-scale compound screens, which are feasible due to the small size of the fish larvae, have already been performed [10]. Two zebrafish models of tauopathies have already been described. Tomasiewicz et al. [11] injected cDNA constructs encoding human TauGFP fusion proteins into zebrafish eggs to study tau pathology. While this approach led to tau phosphorylation at a pathologically relevant epitope in a small number of neurons expressing the injected DNA, it failed to generate transgenic lines with stable and reproducible expression. In addition, tangle formation, disease progression as well as neuronal loss were not described. The generation of stable transgenic lines was first achieved by Bai et al. [12], who described a human tau-transgenic fish using the zebrafish Enolase2 promoter. This promoter allowed expressing tau specifically in the nervous system; however, no signs of tau pathology were observed at larval stages of development. This might be due to insufficient tau protein levels, since high expression levels have been shown to be essential for a concentration-dependent amyloid aggregation mechanism in model systems [13]. We have therefore developed a Gal4-UAS-based expression system to efficiently generate transgenic zebrafish overex- 
pressing high levels of human Tau-P301L or other disease-associated proteins under control of the neuronal zebrafish HuC promoter. The Gal4-UAS system amplifies gene expression by both the strong herpes simplex virus VP16 transcriptional activator, which is fused to Gal4, and the use of 14 UAS sites, to which the Gal4-VP16 binds [14]. The tau-transgenic fish rapidly reproduced pathological signatures of tauopathies already in early developmental stages in a time-dependent manner [15]. Pathological misfolding of tau and hyperphosphorylation at several well-studied epitopes occurred within a few hours of development, and expression of late-stage pathological markers, such as AT8, rapidly progressed over time. Furthermore, NFTs with typical neuropathological features developed after 5 weeks. In addition, the fish displayed abnormal motoneuron function, leading to a retarded escape response. After a few days, they also developed significant neurodegeneration in the nervous system, and the new model allowed for the first time to image the degeneration of neurons live in an animal model by in vivo time-lapse microscopy. Strikingly, taudependent neuronal cell loss was observed well before tangles formed. Thus, our findings may suggest that in contrast to the common opinion, tangles themselves are not neurotoxic but rather soluble tau oligomers. Alternatively, elevated levels of tau monomers might also be toxic, as they may trap cellular proteins and prevent them from executing their physiological functions.

We also used the fish to identify and validate newly developed drugs, which may modify the pathologic hyperphosphorylation of tau. Hyperphosphorylation is believed to be a key initiator of detachment of normal tau from microtubules and subsequent oligomerization and aggregation. Preventing hyperphosphorylation by kinase inhibitors could slow further progression of pathology and thus be an efficient treatment option for AD and FTD [16]. These inhibitors can be developed on a large scale by structure-based design [17], but this approach requires in vivo validation in cell culture and animal models. We validated two newly identified inhibitors of
GSK3, a major kinase in tau phosphorylation [18]. Interestingly, one compound was highly active in preventing abnormal tau phosphorylation, while the other was completely inactive in fish, though equally potent in cell culture. The inactivity of one of the inhibitors could be due to low penetration through the developing blood-brain barrier or rapid degradation or metabolism of the compound. This result illustrates the usefulness of the fish model as an effective in vivo screening tool to quickly and directly identify promising compounds and eliminate substances without reasonable in vivo activity early in the screening process. In addition, the tauopathy-like phenotypes of the transgenic zebrafish may also allow performing unbiased large-scale compound screens to identify new drug targets and new compounds capable not only of reducing tau hyperphosphorylation, but also of restoring axonal transport deficits and preventing synapse dysfunction as well as neuronal cell death.

Since the versatility of the Gal4/UAS-based vector system also allows to quickly subclone and express other proteins associated with neurodegenerative disease, the advantages of the fish system may now also be used to study related disease, such as Parkinson disease or the tau-negative cases of FTD.

Taken together, transgenic zebrafish could be a promising tool to further elucidate the early pathological processes leading synaptic dysfunction in neurodegenerative diseases and also to streamline pharmacological screening for new and effective drugs.

\section{Acknowledgements}

This work was supported by grants from the Deutsche Forschungsgemeinschaft (SFB 596 to B.S. and C.H.; the Center for Integrated Protein Science, to C.H. and B.S., and the Leibniz Award, to C.H.), Elitenetzwerk Bayern and Universität Bayern (to D.P.), and from the European Community's Seventh Framework Programme (FP7/2007-2013) under grant agreement No. 200611 (MEMOSAD, to C.H., B.S., and D.P.). C.H. is supported by a 'Research Professorship' of the LMU.

\section{References}

Transgenic Zebrafish to Study

Tauopathies
1 Haass C, Selkoe DJ: Soluble protein oligomers in neurodegeneration: lessons from the Alzheimer's amyloid beta-peptide. Nat Rev Mol Cell Biol 2007;8:101-112.

2 Grundke-Iqbal I, Iqbal K, Tung YC, Quinlan M, Wisniewski HM, Binder LI: Abnormal phosphorylation of the microtubule-associated protein tau (tau) in Alzheimer cytoskeletal pathology. Proc Natl Acad Sci USA 1986; 83:4913-4917.
3 Kosik KS, Joachim CL, Selkoe DJ: Microtubule-associated protein tau (tau) is a major antigenic component of paired helical filaments in Alzheimer disease. Proc Natl Acad Sci USA 1986;83:4044-4048.

4 Braak H, Braak E: Neuropathological stageing of Alzheimer-related changes. Acta Neuropathol 1991;82:239-259. 
5 Santacruz K, Lewis J, Spires T, Paulson J, Kotilinek L, Ingelsson M, Guimaraes A, DeTure M, Ramsden M, McGowan E, Forster C, Yue M, Orne J, Janus C, Mariash A, Kuskowski M, Hyman B, Hutton M, Ashe KH: Tau suppression in a neurodegenerative mouse model improves memory function. Science 2005; 309:476-481.

6 Hutton M, Lendon CL, Rizzu P, Baker M, Froelich S, Houlden H, Pickering-Brown S, Chakraverty S, Isaacs A, Grover A, Hackett J, Adamson J, Lincoln S, Dickson D, Davies P, Petersen RC, Stevens M, de Graaff E, Wauters E, van Baren J, Hillebrand M, Joosse M, Kwon JM, Nowotny P, Che LK, Norton J, Morris JC, Reed LA, Trojanowski J, Basun H, Lannfelt L, Neystat M, Fahn S, Dark F, Tannenberg T, Dodd PR, Hayward N, Kwok JB, Schofield PR, Andreadis A, Snowden J, Craufurd D, Neary D, Owen F, Oostra BA, Hardy J, Goate A, van Swieten J, Mann D, Lynch T, Heutink P: Association of missense and 5'splice-site mutations in tau with the inherited dementia FTDP-17. Nature 1998;393: 702-705.
7 Götz J, Deters N, Doldissen A, Bokhari L, Ke Y, Wiesner A, Schonrock N, Ittner L: A decade of tau transgenic animal models and beyond. Brain Pathol 2007;17:91-103.

8 Götz J, Ittner L: Animal models of Alzheimer's disease and frontotemporal dementia. Nat Rev Neurosci 2008;9:532-544.

9 Rubinstein AL: Zebrafish: from disease modeling to drug discovery. Curr Opin Drug Discov Devel 2003;6:218-223.

10 Zon LI, Peterson RT: In vivo drug discovery in the zebrafish. Nat Rev Drug Discov 2005; 4:35-44.

11 Tomasiewicz HG, Flaherty DB, Soria JP, Wood JG: Transgenic zebrafish model of neurodegeneration. J Neurosci Res 2002;70: 734-745.

12 Bai Q, Garver JA, Hukriede NA, Burton EA Generation of a transgenic zebrafish model of tauopathy using a novel promoter element derived from the zebrafish eno 2 gene. Nucleic Acids Res 2007;35:6501-6516.

13 Price DL, Sisodia SS, Borchelt DR: Genetic neurodegenerative diseases: the human illness and transgenic models. Science 1998; 282:1079-1083.
14 Koster RW, Fraser SE: Tracing transgene expression in living zebrafish embryos. Dev Biol 2001;233:329-346.

15 Paquet D, Bhat R, Sydow A, Mandelkow E, Berg S, Hellberg S, Fälting J, Distel M, Köster RW, Schmid B, Haass C: A zebrafish model of tauopathy allows in vivo imaging of neuronal cell death and drug evaluation. J Clin Invest 2009; 119:1382-1395.

16 Mazanetz MP, Fischer PM: Untangling tau hyperphosphorylation in drug design for neurodegenerative diseases. Nat Rev Drug Discov 2007;6:464-479.

17 Bhat R, Xue Y, Berg S, Hellberg S, Ormo M, Nilsson Y, Radesater AC, JerningE, Markgren PO, Borgegard T, Nylof M, Gimenez-Cassina A, Hernandez F, Lucas JJ, Diaz-Nido J, Avila $\mathrm{J}$ : Structural insights and biological effects of glycogen synthase kinase 3-specific inhibitor ar-a014418. J Biol Chem 2003;278:4593745945.

18 Doble BW, Woodgett JR: Gsk-3: tricks of the trade for a multi-tasking kinase. J Cell Sci 2003;116:1175-1186. 